\title{
Type A Behavior: An Ecological Approach
}

\author{
Lewis H. Margolis, ${ }^{1}$ Kenneth R. McLeroy, ${ }^{2}$ \\ Carol W. Runyan, ${ }^{3}$ and Berton H. Kaplan ${ }^{3,4}$
}

Accepted for publication: November 30, 1982

The recognized association between Type $A$ behavior and coronary heart disease has prompted efforts to alter the behavior's deleterious components in both individuals who have experienced myocardial infarction and those who are at risk for that disease. Utilizing concepts from psychology, sociology, history, and other disciplines and material from scholarly and popular literature, this paper suggests that instead of singularly concentrating on components at the level of the individual, it is important to view Type $A$ behavior from an ecological perspective, with attention directed at the interpersonal, institutional, and cultural environments of individuals. This perspective will enhance understanding of Type $A$ behavior and possibly stimulate interventions at the primary as well as secondary and tertiary prevention levels.

KEY WORDS: Type A behavior; ecology; etiology.

\section{INTRODUCTION}

This nation's interest in health is focusing increasingly on life-style explanations for the etiology of illness (U.S. Department of Health,

The coauthors would like to thank Brenda DeVellis, Michel Ibrahim, Lynda Powell, Meyer Friedman, and two anonymous reviewers for comments on an early draft of the manuscript. The Bush Institute for Child and Family Policy of the University of North Carolina provided support for the first and third coauthors and the Research Triangle Institute provided support for the second coauthor.

"University of Michigan.

${ }^{2}$ University of North Carolina at Greensboro.

${ }^{3}$ University of North Carolina at Chapel Hill.

${ }^{4}$ Correspondence to Dr. Kaplan, Department of Epidemiology, Rosenau Hall, University of North Carolina, Chapel Hill, North Carolina 27514. 
Education and Welfare, 1979; Select Panel for the Promotion of Child Health, 1981). Clinical investigators have directed substantial efforts at understanding the role of life-style in health because concentration on traditional risk factors has provided incomplete understanding of most morbidity and mortality. Similarly, policymakers have turned to life-style explanations of illness because of their need to allocate scarce resources in an economic environment where the costs of conventional care are rising uncontrollably. The hope is that by understanding and then altering deleterious life-styles or behaviors, a more healthy population will result. Consequently, the established association between Type A behavior and coronary heart disease has appeal for both health professionals and policymakers alike. If the developmental precursors and determinants of the behavior pattern can be identified, it will be possible to implement preventive efforts at a variety of intervention levels, which will lead to a reduction in coronary heart disease, the leading cause of premature death in the United States.

As the Review Panel on Coronary-Prone Behavior and Coronary Heart Disease has described, substantial efforts have been devoted to assessing Type A behavior, probing its physiological mechanisms and implementing intervention trials (Review Panel on Coronary-Prone Behavior and Coronary Heart Disease, 1981). The panel also notes that efforts to investigate both the developmental and the cultural aspects of Type A behavior are inchoate. Therefore, in posing questions about the next stage of research, the panel suggests that "If Type A behavior is conceptualized as the result of a predisposition stimulated by appropriate environmental challenges, then it would appear appropriate to divide the assessment tasks into three components: the relevant personality predisposition, the challenges and demands emanating from the person's various environmental life settings, and the actual behavior and reactions that are anticipated when these demands activate the predisposition" ( $p$. 1205).

Building upon the Panel's recognition of the interactions between environments and the individual, the purpose of this paper is to analyze Type A behavior within an "ecological" model, such as that recently proposed by Bronfenbrenner for the study of child development (1979). This model permits the examination of how environments at different levels-interpersonal, institutional, and cultural-promote Type A behavior and how these environments may influence the development of this behavior in children. Furthermore, the model provides a mechanism for formulating propositions about how these environments and the individual interrelate in the development of Type A behavior. Selected examples of propositions are offered throughout the discussion. 


\section{THE ECOLOGICAL MODEL}

As with many health phenomena, the conceptualization of Type A behavior resulted from clinical observations of individual patients by physicians. Beginning with individuals who had experienced myocardial infarction, Friedman looked retrospectively and identified behavioral characteristics shared by these patients (Friedman and Rosenman, 1974). From those observations, the well-known characteristics which comprise Type A behavior have emerged-excessive competitive achievement, time urgency, aggressiveness, and hostility.

Consistent with epidemiological principles, the description of Type A behavior has been further refined through the use of cohort studies (Jenkins et al., 1974) and then intervention trials (Thoresen et al., 1981). Although some investigators have noted cultural variations in both coronary artery disease and Type A behavior (Cohen et al., 1979), studies in general have focused on individual characteristics.

Concentrating on individual characteristics limits understanding of the relationship between Type A behavior and coronary heart disease. To elaborate the nature of Type A behavior and explicate the role of Type A characteristics in both health and illness, it is necessary to assume a perspective that incorporates numerous levels of human experience. Recently, Bronfenbrenner (1979) and others (Jenkins, 1979; Radley, 1982) have presented ecological models which are applicable to the issue of Type A behavior. In the Bronfenbrenner model, the environments in which children develop are presented as a set of nested situations. On the interpersonal level are the "activities, roles and interpersonal relations experienced in a given setting" (p. 22). For young children this setting is most often the family, but for adolescents or adults this may be any of the primary settings where activities, roles, and interpersonal relations are experienced. For example, the work place as well as the family is part of the adult's interpersonal level. School and family are both part of the adolescent's environment.

On the institutional level are the "social structures, both formal and informal, that do not themselves contain the developing person but impinge upon or encompass the immediate settings in which the person is found and thereby influence what goes on there" (p. 25). In considering Type A behavior, the most influential structure is that of work or, more generally, the system of economic relationships within society.

On the cultural level are the values and ideologies which underlie relationships and activities on the other levels of this model. Among others, these values include cultural definitions of the individual and the group. 
In order to comprehend the emergence of Type A behavior, it is instructive to consider each of these levels of analysis as well as the intrapersonal characteristics or individual traits. Using this multilayered, ecological model, the development of Type A behavior is analyzed.

\section{Intrapersonal Level}

Understanding of some of the mechanisms involved in Type A behavior emerges from the discipline of social psychology - specifically, the areas of achievement striving (Snow, 1978) and competitiveness (Gotay, 1981). However, the psychological processes underlying these behaviors and their relative importance in disease pathophysiology are less well understood. The purpose of this section is to suggest some of the psychological processes which may underlie Type $\mathrm{A}$ behavior.

Intense achievement striving as one of the characteristics of Type A behavior implies social comparison processes. Often achievement involves making a comparison of one's performance to some standard. This standard can be the performance of others, one's own previous performance, or some ideal standard. While it is currently unclear exactly which standards are chosen by individuals expressing Type A behavior, it is apparent that they tend to adopt very high performance standards. Moreover, choosing a high performance standard in multiple tasks results in individuals' rarely being able to meet all of their performance requirements and consequently always striving to improve. It may be, in fact, that the Type A individual cannot decide on an explicit performance standard and must therefore be constantly striving to improve since no performance is ever satisfactory.

Attributional processes may also relate to the development of Type A behavior. An attribution refers to an individual's explanation of the causes of an event and, therefore, affects expectations about the future. There are several dimensions of attributions which are of importance for understanding Type A behavior. These include (1) the locus of causality, (2) stability, and (3) generality (Abramson et al., 1980).

Locus of causality refers to attributions made to either internal or external factors. For example, the attribution of outcomes to either effort or ability represents the assignment of cause to internal factors, whereas the attribution to task difficulty represents the assignment of cause to external factors. Among the internal factors, attributions to effort rather than ability are fundamental to Type A behavior. If individuals attribute positive outcomes to effort rather than ability, and they have high achievement standards, they will tend to exert greater effort in performancerelated tasks than individuals who attribute outcomes to ability. This may explain the finding that on laboratory tasks with random 
outcomes, Type A individuals exert effort of greater magnitude and duration and have higher expectations about the positive benefits of their efforts than Type B individuals (Glass and Carver, 1980).

The stability dimension refers to the extent to which the attributed causes are stable over time. In the effort versus ability attribution described above, ability represents a stable attribution, while effort represents an unstable attribution. Other performance-related attributions include task difficulty which is stable or luck which is unstable (Weiner and LitmanAdizes, 1980).

The generality dimension refers to whether one perceives causes as global or specific. The more one attributes outcomes to specific circumstances, the more uncertain the future. If Type $A$ individuals tend to view circumstances as specific rather than global, then they will find it necessary to be eternally vigilant to prevent negative outcomes or to achieve positive ones.

Social learning theory (Bandura, 1977) suggests that individuals develop expectations about the outcomes of future events based on their previous performances, through observation of the performances of others, or by experiencing the performances of others through communication. However, expectations about the future are not just a result of the link between performance and outcomes but also depend upon attributional processes. For example, Type A individuals would be expected to overestimate the extent to which positive outcomes are under behavioral control while underestimating the probability that positive outcomes will occur without the exertion of effort (Snow, 1978).

Proposition: Individuals with Type A behavior have higher
performance standards than other individuals.
Proposition: Individuals with Type A behavior tend to make
attributions to effort rather than ability and to
expect future outcomes to be based on intense effort
rather than ability.

\section{Interpersonal Level}

A number of interpersonal processes appear to elicit and maintain Type A behavior. These interpersonal processes include high expectations and aspirations on the part of salient others, unclear, inadequate, or negative performance feedback, and unrealistic performance feedback by others.

Type A behavior seems to represent a long-term pattern of responses to competitive situations which is initiated early in life and which is characterized at the interpersonal level by the self-selection into and initiation of competitive situations. Furthermore, this behavior appears to 
develop through interactions with significant others who place high expectations on the child and who reward aggressive, competitive behavior. Matthews (1977) has suggested that the escalation of performance standards by parents may play an important part in the etiology of Type A behavior, for example, by encouraging achievement striving. As noted by Cohen et al. (1979), associations have been demonstrated between achievement striving on the one hand and high parental expectations and aspirations, frequent approval and disapproval, competitiveness, and authoritarian discipline techniques on the other. Cohen et al. also note that aggressive behavior is exhibited by children when salient models are aggressive and when aggression is rewarded and returned in the home. Gotay (1981) found that Type A college students predicted that they would win more money in a competitive situation than their opponents, but anticipation of winning was not different in a cooperative situation.

Further understanding of the role of interpersonal processes in the development of Type A behavior comes from examining differences in socialization processes of boys and girls. As Dweck and Licht (1980) point out, boys are typically taught to attribute their failures to not having tried hard enough and are admonished to "work harder next time." Girls, on the other hand, attribute their successes to effort and their failures to lack of ability. When faced with the disappointments of failing, girls are more likely than boys to give up, instead of struggling to meet future challenges. Matthews (1977) and Waldron (1978) suggest that this pattern, established early in life, may in part explain some of the sex differences seen in both child and adult Type A behavior.

While aggressive, competitive interpersonal situations appear to promote and elicit Type A behavior in some individuals, the behavior also influences the nature, structure, and content of interpersonal relationships. That is, there is reciprocity between the behavior of individuals and the context in which it occurs. It appears that Type A individuals not only may select competitive situations but also may initiate competition in otherwise noncompetitive situations. This may have unanticipated consequences on the nature of their interpersonal relationships. For example, we suggest that individuals exhibiting Type A behavior are more likely to define their intimate interpersonal relationships as competitive. They also may be more likely to have less intense relations and have greater difficulty with interpersonal intimacy.

In addition to parental influences are the influences of wider social networks. As Hamburg and Killilea (1979) have suggested, social networks consist of the interpersonal connections or linkages between an individual and other individuals with whom relationships of varying intimacy are 
maintained. Network properties include (1) the number of persons, (2) the intensity of the relationships, (3) the extent to which members share relationships with each other, (4) the similarity in life-style and values between network members, (5) the extent to which members have face-toface contacts or live within a geographic locale, and (6) the degree of complementarity or mutuality of the relationships.

Type A behavior may affect the social networks in which individuals are involved and the networks may in turn affect the initiation and maintenance of Type A behavior. For example, individuals with Type A behavior may be more likely to have larger, less dense, weaker, more dispersed, less homogeneous, and less reciprocal social networks. The emphasis on achievement may result in Type A individuals receiving less emotional support than individuals with less pronounced Type A behavior. However, the level of satisfaction with the amount of emotional support received may not vary between Type $\mathrm{A}$ individuals and others.

\section{Proposition: Sex role socialization contributes to the male/female differences in the prevalence of Type A behavior. \\ Proposition: Network structures for individuals with Type \\ A behavior will differ from those for individuals without Type $\mathrm{A}$ behavior. \\ Proposition: Type A individuals tend to select competitive, rather than cooperative, performance situations.}

\section{Institutional Level}

The intrapersonal and interpersonal processes described above are nested within institutional frameworks. Examination of institutions or social structures involving work, education, and recreation reveal numerous influences upon Type A behavior. For example, after characterizing certain work environments as Type A or Type B, Matteson and Ivancevich (1982) showed that Type A individuals in Type A organizations tended to report the most negative health outcomes. Similarly, Mettlin (1976) has demonstrated an association between the probability of an individual's having Type A behavior and work characteristics such as employer expectations, rate of change in income over 10 years, and number of subordinates.

Basically, institutions within society have incorporated at least four characteristics which seem promotive of Type A behavior: (1) reward 
systems that foster aggressive competition and achievement striving; (2) limited controllability and/or predictability of success or failure, accompanied by little tolerance for error; (3) numerous role demands, resulting in both time and opportunity conflicts; and (4) time demands that encourage time-urgent and/or aggressive behavior.

Reward systems vary across institutions, but from spelling bees to Little League to grading on the curve, competition is emphasized to young people. Students are taught that to get ahead in the world, they must continually try harder and that through successful competition one can "live up to one's potential." Generally, when the limits of competition are clear and potentially reachable, they may be less promotive of Type A behavior than when goals and standards are ambiguous. Unfortunately, questions such as how many publications are sufficient for promotion and how good a grade point average is necessary for the right job are rarely explicit.

Corporate managers are another example of people for whom the need for control is intense and yet their abilities are constrained. The uncertainty provoked by such phenomena as inflation and foreign political upheaval undermines the capabilities of even the most talented managers.

Time demands on the developing student or employee also are related to institutional characteristics and may contribute to Type A behavior. Productivity is highly valued and is measured in terms of units of output per time. Reminders of the passage of time include school bells, time clocks, and repeated, crucial deadlines. While there is much waste of some resources in our society, the wasting of one's time is not tolerated.

Examination of the effects of institutional demands on women provides additional insight into the role of these environments in the development of Type A behavior. As women enter the work place in growing numbers, they must contend with role and time pressure conflicts which are more excessive than those which men face. Since women still assume the preponderance of household responsibilities, there is tension between family responsibilities on the one hand and work responsibilities on the other. This overriding tension is then exacerbated by the fact that there are multiple roles and responsibilities within both the home and the work place.

A second potential source of stimulation of Type $A$ behavior for women stems from job discrimination. The occurrence of discrimination produces aggressive achievement striving because women must work that much more intensely just to attain or maintain equal status with male counterparts. Similarly, discriminatory standards promote a lack of predictability and controllability.

While socialization processes described at the interpersonal level favor a reduced tendency for women to adopt Type $\mathbf{A}$ behavior, institutional 
environments promote this behavior. As the influences of the work place are better understood, institutions hopefully will change in order to alleviate the stimuli for Type A behavior for both women and men (Ibrahim, 1980).

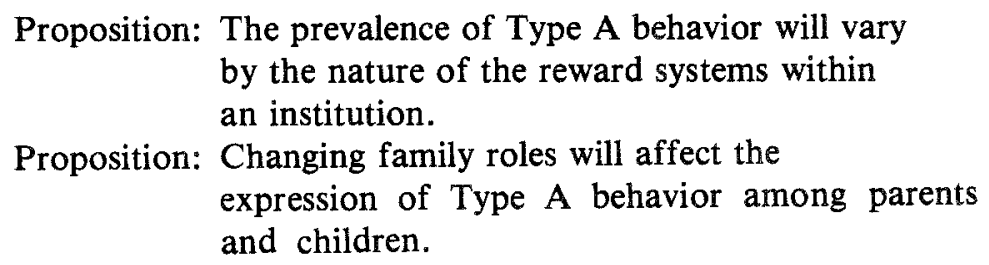

\section{Cultural Level}

The third environment in which individuals function is cultural. For the purposes of this discussion, culture refers to the values and practices which characterize the responses of groups of people to three general questions or problems: (1) What is the relationship of the individual to the group? (2) What is the relationship of the individual to the universal, such as history or a supreme being? and (3) What is the perception or meaning of time? In turning to the cultural answers to these questions, it becomes apparent that Western civilization has provided a value structure which promotes excessive competitive achievement, time urgency, aggressiveness, and hostility.

Examination of contemporary American culture provides perspective on the development of Type A behavior. Lasch (1979) has commented on the growing complexity and consequent social disintegration in The Culture of Narcissism. In viewing the relationship of the individual to the group, Lasch cites developments in family life, relationships between sexes, education, business, and sports to argue that preoccupation with the self has not led to an easing of tensions, a heightened awareness, and an enhancement of desire. Instead, the narcissist "demands immediate gratification and lives in a state of restless, perpetually unsatisfied desire" (p. 23). In other words, self-indulgence has intensified anxiety because the intense need for approval and acclaim, in the end, precludes individuals from finding fulfillment with other individuals. Driven by the need for approval, the narcissist views other individuals as competitors for that approbation. Relationships of all types-personal, parental, work, and others - become combative instead of mutually supportive.

The descent into narcissism and the resultant combativeness of relationships further exacerbate Type A behavior since it is occurring in the midst of rapid change. As Toffler (1970) has explained, transience characterizes relationships to things, places, people, organizations, and ideas. This decreasing duration of relationships has increased the element of 
uncertainty in all aspects of life. Complicating the shortened duration of relationships is the astounding rate at which new ideas and things become part of contemporary life, a process which further heightens uncertainty. Toffler contends that the major task of both individuals and societies is to develop the ability to adapt to change. Decisional stress, as Toffler calls it, combines with sensory and cognitive overload to produce maladaptive behavior and ilness. Type A behavior and resultant coronary heart disease are prime examples.

The origins of Type A behavior are found in classical Greek culture, where the definition of the individual demonstrates a concern for certain Type A characteristics, if not the clearly defined behavior. Kitto (1954) suggests that the one characteristic which most distinguished Greek society from others was the concept of freedom. In contrast to the members of despotic societies, the Greek viewed themselves as linked to and dependent upon the polis, but also as individual citizens whose rights as individuals were guaranteed by the polis. The Greek individual is further defined by the concept of arete or excellence, which represented the achievement of mastery by the whole person, not just specialized parts. Greek heroes such as Odysseus or Achilles were skilled warriors but talented speakers and poets as well. However, counterbalancing the drive to achieve mastery was an awareness of the flaw of hybris, overreaching or excessive pride. In defining the relationship of the individual to the universal, philosophers admonished the Greeks to "know thyself," meaning to understand the limits of one's abilities. Repeatedly, the Greek tragedians grappled with the dangers inherent in attempting to reach from the realm of mortals into the realm of the gods.

Max Weber's (1958) classic, The Protestant Ethic and the Spirit of Capitalism, articulates responses to the questions posed above and thus provides additional historical insight into the emergence of Type $A$ behavior. It is an economic fact that the desire to accumulate goods is essential to the rise of capitalism. According to Weber, Protestantism, or more precisely Calvinism, provided the ethical basis which enabled people not only to justify their accumulation of worldly goods but also to rationalize the differences between individuals. Work-and its physical manifestation, the accumulation of capital-was considered a form of prayer. Work represented the means by which individuals pursued the task or calling that God had established for them. In addition, since an individual life span was viewed as infinitesmal in duration in relationship to the divine time frame, work served another vital function in that it prevented individuals from yielding to other temptations, which would have diverted them from the pursuit of their callings.

Central to the notion of Type A behavior is the Calvinist idea that it is necessary to continue accumulating goods in excess of one's needs. Weber's 
analysis demonstrates the individual's competitive achievement and sense of time urgency on a cultural level. The belief that work is a manifestation of being "saved" and that salvation is always uncertain promotes the unclear performance expectations, social comparisons with high achievers, and the lack of clear links between efforts and outcomes which are components of the intrapersonal and interpersonal perspectives on Type $A$ behavior.

In order to understand better the role of culture in promoting Type A behavior, it is enlightening to examine its development in non-Western cultures. Initial attempts have been made to study Type A behavior among the Japanese (Cohen et al., 1979; Matsumoto, 1970). Japan is a highly industrialized, urbanized society, which, however, is based on cultural values which differ markedly from those of the West. For example, the Japanese and Western concepts of the individual's relationship to the group are different. In the West, children are viewed as dependent organisms which must learn independence during the course of development. In Japan, children are considered independent organisms which must be drawn into increasingly dependent relationships in order to develop (Vogel and Vogel, 1968). This perception of participation in the group is reflected linguistically in the infrequent use of the pronoun "I" among the Japanese.

The fact that the Japanese have been characterized as "hard working" as opposed to "hard driving" has been postulated as one reason for the relatively low rate of coronary heart disease (Cohen et al., 1979). Dependence on others may stimulate individuals to work hard for the benefit of the group, whereas independence promotes hard-driving, self-reliant behavior. The cultivation of dependence which lessens hard-driving behavior may also counter social disintegration, which seems more severe in the West than in Japan. The anxiety-producing combativeness of which Lasch writes is simply absent in Japanese culture. The disorienting change articulated by Toffler is also deflected by the Japanese dependence because the family or the group, by its nature, assures relationships of longer duration than the transient ones of individuals.

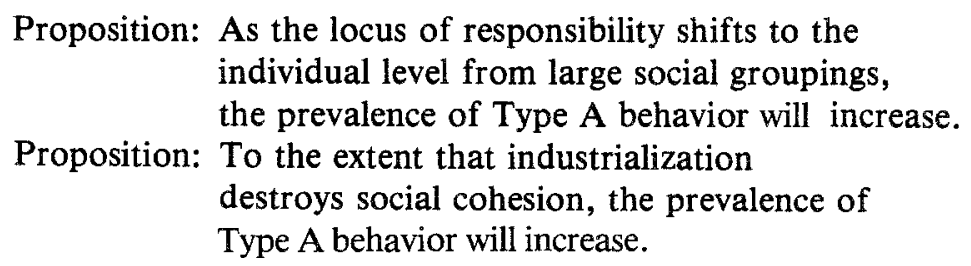

\section{CONCLUSIONS}

In order to increase understanding of both Type $\mathrm{A}$ behavior and its role in the pathogenesis of coronary heart disease, an ecological framework 
Table I. Ecological Levels of Analysis of Type A Behavior

\begin{tabular}{ll}
\hline Environment & \multicolumn{1}{c}{ Contributing factors } \\
\hline Intrapersonal & $\begin{array}{l}\text { Unrealistic social comparison processes } \\
\text { Attributional dimensions of locus of control in which high achieve- } \\
\text { ment orientation is combined with unstable (effort rather than } \\
\text { ability), internal (rather than external), and specific (rather than } \\
\text { global) attributions }\end{array}$ \\
& High expectations by salient others (achievement striving) \\
Unclear performance feedback & Reward for aggressive, competitive behavior \\
& Diffuse, heterogeneous, dispersed social networks \\
& Reward systems which foster aggressiveness \\
Institutional & Limited controllability of success or failure \\
& Numerous role demands \\
& Numerous time demands \\
& Individualistic orientation toward causality combined with devalua- \\
tion of individuals & Time orientation \\
Cultural & Cultural complexity \\
& Rapid rates of cultural change \\
\hline
\end{tabular}

is useful. Intrapersonal characteristics such as excessive, competitive achievement, time urgency, and hostility are expressed within nested environments of increasing complexity, as shown in Table $I$.

Within each level, a variety of mechanisms contributes to the promotion of Type A behavior. Consequently, it is possible to formulate, implement, and evaluate intervention strategies for each level. Until the present time, most interventions have attempted to alter intrapersonal or interpersonal aspects, particularly in those individuals who already suffer from coronary heart disease (Thoresen et al., 1981).

Proposition: Interventions directed at more than one environmental level are more likely to be successful than those directed at a single level.

Proposition: Interventions directed at only one environmental level may fail because of influences from other levels.

In traditional public health terms, many strategies have been either tertiary or secondary prevention (Runyan et al., 1982). Examples of the former include interventions directed at altering Type $\mathrm{A}$ behavior in those individuals who already suffer from coronary heart disease. The latter strategies are directed at diminishing Type $\mathbf{A}$ behavior in those individuals who manifest it but who have not yet experienced coronary disease. 
An ecological framework, in considering the multilayered, environmental mechanisms which promote Type A behavior, enables practitioners and policymakers to devise and implement primary prevention strategies at all levels. Such strategies, in attempting to understand and alter the mechanisms responsible for Type A behavior, would prevent or at least ameliorate its development. As with many public health problems, primary prevention may be the most efficient and equitable strategy for alleviating both Type A behavior and coronary heart disease.

\section{REFERENCES}

Abramson, L. Y., Garber, J, , and Seligman, M. E. P. (1980). Learned helplessness in humans: An attributional analysis. In Garber, J., and Seligman, M. E. P. (eds.), Human Helplessness: Theory and Applications, Academic Press, New York, pp. 3-34.

Bandura, A. (1977). Social Learning Theory, Prentice-Hall, Englewood Cliffs, NJ.

Bronfenbrenner, U. (1979). The Ecology of Human Development, Harvard University Press, Cambridge, Mass.

Cohen, J. B., Syme, S. L., Jenkins, C. D., Kagan, A., and Zyzanski, S. J. (1979). Cultural context of type A behavior and risk for CHD: A study of Japanese-American males. $J$. Behav. Med. 2:375-384.

Dweck, C., and Licht, B. (1980). Learned helplessness and intellectual achievement. In Garber, J., and Seligman, M. E. P. (eds.), Human Helplessness: Theory and Applications, Academic Press, New York, pp. 197-221.

Friedman, M., and Roseman, R. H. (1974). Type A Behavior and Your Heart, Alfred Knopf, New York.

Glass, D. C., and Carver, C. S. (1980). Helplessness and the coronary prone behavior pattern. In Garber, J., and Seligman, M. E. P. (eds.), Human Helplessness: Theory and Aplications, Academic Press, New York, pp. 223-243.

Gotay, C. C. (1981). Cooperation and competition as a function of type A behavior. Personal. Soc. Psychol. Bull. 7: 386-392.

Hamburg, D. A., and Killilea, M. (1979). Relation of social support, stress, illness, and use of health services. In Healthy People: The Surgeon General's Report on Health Promotion and Disease Prevention (background papers), DHEW (PHS) No. 79-55071A. U. S. Government Printing Office, Washington, D.C., pp. 253-276.

Ibrahim, M. A. (1980). The changing health status of women. Am. J. Publ. Health 70: 120-121.

Jenkins, C. D. (1979) Psychosocial modifiers of response to stress. J. Human Stress 5: 315.

Jenkins, C. D., Rosenman, R. H., and Zyzanski, S. J. (1974). Prediction of clinical coronary heart disease by a test for coronary-prone behavior. New Engl. J. Med. 290:1271-1275.

Kitto, H. D. F. (1954). The Greeks, Penguin Books, Baltimore.

Lasch, C. (1979). The Culture of Narcissism, W. W. Norton, New York.

Matsumoto, Y. S. (1970). Social stress and coronary heart disease in Japan: A hypothesis. Milbank Mem. Fund Q. 48: 9-36.

Matteson, M. T., and Ivancevich, D. B. A. (1982). Type A and B behavior patterns and self-reported health symptoms and stress: Examining individual and organizational fit. J. Occup. Med. 24: 585-589.

Matthews, K. (1977). Caregiver-child interactions and the type A coronary prone behavior pattern. Child Dev. 48:1752-1756.

Mettlin, C. (1976). Occupational careers and the prevention of coronary-prone behavior. Soc. Sci Med. 10: 367-372. 
Review Panel on Coronary-Prone Behavior and Coronary Heart Disease (1981). Coronaryprone behavior and coronary heart disease: A critical review. Circulation 63: $1199-1215$.

Radley, A. R. (1982). Theory and data in the study of coronary proneness. Soc. Sci. Med. 16: $107-114$.

Runyan, C. W., DeVellis, R. F., DeVellis, B. M., and Hochbaum, G. M. (1982). Health psychology and the public health perspective: In search of the pump handle. Health Psychol. 1: 169-180.

Select Panel for the Promotion of Child Health (1981). Better Health For Our Children: A National Strategy, DHHS (PHS) No. 79-55071, U.S. Government Printing Office, Washington, D.C.

Snow, B. (1978). Level of aspiration in coronary prone and non coronary prone adults. Personal. Soc. Psychol. Bull. 4: 416-419.

Thoresen, C. E., Telch, M. J., and Eagleston, J. R. (1981). Approaches to altering the type A behavior pattern. Psychosomatics 22: 472-482.

Toffler, A. (1970). Future Shock, Random House, New York.

United States Department of Health, Education and Welfare (1979). Healthy People: The Surgeon General's Report on health Promotion and Disease Prevention, DHEW (PHS) No. 79-55071, U.S. Government Printing Office, Washington, D.C.

Vogel, E., and Vogel, S. H. (1968). The patterning of emotions. In Geiger, H. K. (ed.), Comparative Perspectives on Marriage and the Family, Little, Brown, Boston, pp. 68-77.

Waldron, I. (1978). Sex differences in the coronary prone behavior pattern. In Dembroski, T.M., Weiss, S. M., Shields, J. L., Haynes, S., and Feinleib, M. (eds.) CoronaryProne Behavior, Springer-Verlag, New York.

Weber, M. (1958). The Protestant Ethic and the Spirit of Capitalism, Charles Scribner's Sons, New York.

Weiner, B., and Litman-Adizes, T. (1980). An attributional expectancy-value analysis of learned helplessness and depression. In Garber, J., and Seligman, M. E. P. (eds.), Human Helplessness: Theory and Applications, Academic Press, New York, pp. 35-57. 